\title{
Antimicrobial activity of octenidine against multidrug-resistant Gram-negative pathogens
}

\author{
R. Alvarez-Marin ${ }^{1,2,3,4}$ - M. Aires-de-Sousa ${ }^{5}$ - P. Nordmann ${ }^{1,2,3,6}$ - N. Kieffer ${ }^{1,2,3}$. \\ L. Poirel ${ }^{1,2,3}$
}

\begin{abstract}
Multidrug-resistant (MR) Gram-negative (GN) pathogens pose a major and growing threat for healthcare systems, as therapy of infections is often limited due to the lack of available systemic antibiotics. Well-tolerated antiseptics, such as octenidine dihydrochloride (OCT), may be a very useful tool in infection control to reduce the dissemination of MRGN. This study aimed to investigate the bactericidal activity of OCT against international epidemic clones of MRGN. A set of five different species (Escherichia coli, Klebsiella pneumoniae, Enterobacter cloacae, Acinetobacter baumannii, and Pseudomonas aeruginosa) was studied to prove OCT efficacy without organic load, under "clean conditions" ( $0.3 \mathrm{~g} / \mathrm{L}$ albumin $)$ and under "dirty conditions" ( $3 \mathrm{~g} / \mathrm{L}$ albumin $+3 \mathrm{~mL} / \mathrm{L}$ defibrinated sheep blood), according to an official test norm (EN13727). We used five clonally unrelated isolates per species, including a susceptible wild-
\end{abstract}

L. Poirel

laurent.poirel@unifr.ch

$1 \quad$ Emerging Antibiotic Resistance Unit, Medical and Molecular Microbiology, Department of Medicine, Faculty of Science, University of Fribourg, rue Albert Gockel 3, 1700 Fribourg, Switzerland

2 French INSERM European Unit, University of Fribourg (LEA-IAME), Fribourg, Switzerland

3 National Reference Center for Emerging Antibiotic Resistance (Switzerland), Fribourg, Switzerland

4 Hospital Universitario Virgen del Rocio y Virgen Macarena, Seville, Spain

5 Escola Superior de Saúde da Cruz Vermelha Portuguesa, Lisbon, Portugal

6 University of Lausanne and University Hospital Center, Lausanne, Switzerland type strain, and four MRGN isolates, corresponding to either the $3 \mathrm{MRGN}$ or $4 \mathrm{MRGN}$ definition of multidrug resistance. A contact time of $1 \mathrm{~min}$ was fully effective for all isolates by using different OCT concentrations $(0.01 \%$ and $0.05 \%)$, with a bacterial reduction factor of $>5 \log _{10}$ systematically observed. Growth kinetics were determined with two different wild-type strains (A. baumannii and K. pneumoniae), proving a time-dependent efficacy of OCT. These results highlight that OCT may be extremely useful to eradicate emerging highly resistant Gram-negative pathogens associated with nosocomial infections.

\section{Introduction}

Multidrug-resistant (MR) Gram-negative (GN) bacteria pose a major and growing threat for healthcare systems, given the paucity of available and efficient antibiotics drastically complicating the treatment of infections [1, 2]. MR Enterobacteriaceae, Pseudomonas, and Acinetobacter strains have emerged as particularly serious concerns [3, 4].

Antiseptic molecules may help to reduce the dissemination of MR bacteria, especially in high-risk areas, such as intensive care units, when used in patient decolonization procedures [5, 6]. However, reduced susceptibility of some MRGN isolates to some frequently used biocides (i.e., chlorhexidine) has been reported [7-9]. A significant bactericidal activity of octenidine dihydrochloride (OCT) was demonstrated against mupirocinresistant methicillin-resistant Staphylococcus aureus (MRSA) strains [10]. However, information about its efficacy against MRGN remains poorly investigated [11, 12]. In the present study, we aimed to assess the in vitro bactericidal activity of OCT against the most relevant GN species responsible for hospital-acquired infections, including isolates exhibiting MR phenotypes. 


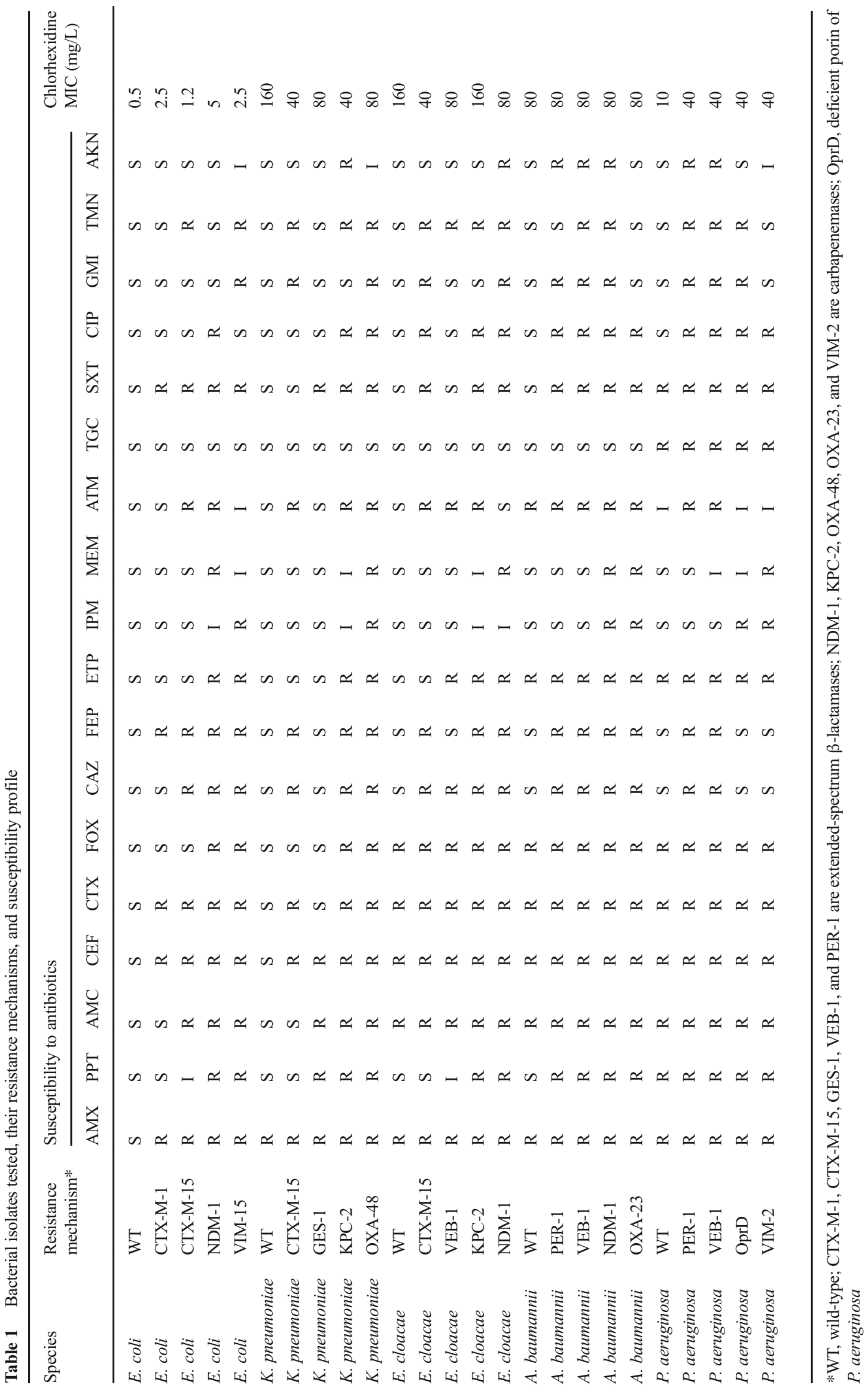




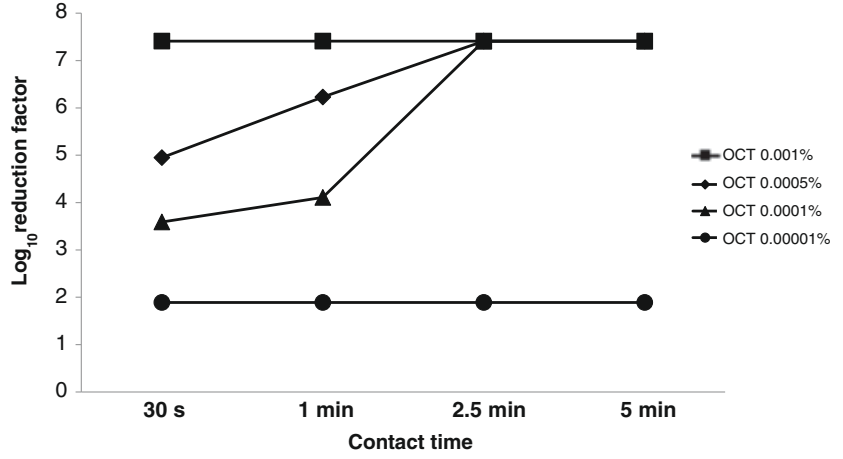

Fig. 1 Time-dependent efficacy of different concentrations of octenidine against Acinetobacter baumannii wild type. These data are representative of the efficacy observed for the other strains tested

\section{Methods}

We selected bacterial isolates from five different clinically relevant GN species (Escherichia coli, Klebsiella pneumoniae, Enterobacter cloacae, Acinetobacter baumannii, and Pseudomonas aeruginosa) from the Culture Collection of the Emerging Antibiotic Resistance Unit (University of Fribourg, Switzerland). Five clonally unrelated isolates were chosen for each species, including, in each case, a single susceptible wild-type strain, and four MR isolates previously characterized for their sequence type and resistance mechanisms, including the production of extended-spectrum $\beta$-lactamases (ESBLs) and carbapenemases (Table 1). Susceptibility to antibiotics was determined by disk diffusion, according to European Committee on Antimicrobial Susceptibility Testing (EUCAST) breakpoints [13]. All the clinical isolates corresponded to either 3MRGN (resistance to three out of the following antibiotic substances: penicillins, cephalosporins, quinolones, and carbapenems) or 4MRGN (resistance to all four pre-cited classes), according to the Robert Koch Institute definition of multidrug resistance [14].

Evaluation of the minimum inhibitory concentrations (MICs) of chlorhexidine digluconate (CHG; Sigma-Aldrich, St. Louis, MO, USA) were carried out, following the standards of the Clinical and Laboratory Standards Institute
(CLSI) [15]. The range of concentrations tested was 0.3$312.8 \mathrm{mg} / \mathrm{L}$, as used previously [10].

The efficacy of OCT was determined in accordance with BS EN 13727:2012+A1 [16], (a) without organic load, (b) under "clean conditions" ( $0.3 \mathrm{~g} / \mathrm{L}$ BSA, Sigma-Aldrich, St. Louis, MO, USA), and (c) under "dirty conditions" ( $3 \mathrm{~g} / \mathrm{L}$ BSA $+3 \mathrm{~mL} / \mathrm{L}$ defibrinated sheep blood, Oxoid, Pratteln, Switzerland). Samples were incubated for different contact times (30 s, 1, 2.5, or $5 \mathrm{~min}$ ) with OCT (Schülke \& Mayr $\mathrm{GmbH}$, Germany) diluted to the final test concentrations ranging from $0.00001 \%$ to $0.01 \%$. After the given contact times, the activity of OCT was neutralized using a combination of $0.1 \%$ tryptone, $0.85 \% \mathrm{NaCl}, 3 \%$ Tween $80,0.3 \%$ lecithin, $3 \%$ saponin, and $0.1 \%$ histidine, without interfering with bacterial growth. Subsequently, serial dilutions of the final mixture were spread onto neutralizing agar plates and incubated for $24 \mathrm{~h}$ and $48 \mathrm{~h}$ at $37^{\circ} \mathrm{C}$. Colonies were counted and the reduction factor (RF) was determined as the difference between the $\log _{10}$ number of cells in the test solution at the beginning of the contact time and the $\log _{10}$ number of recovered colonies in the test solution. A $5 \log _{10}$ reduction within $\leq 5$ min was considered effective according to the test norm EN13727.

\section{Results and discussion}

Although all E. coli isolates had low MICs (ranging from 1 to $4 \mathrm{mg} / \mathrm{L}$ ), all other isolates had MICs of CHG ranging from 32 to $128 \mathrm{mg} / \mathrm{L}$, thus showing poor efficacy of the molecule against most MRGN isolates (Table 1). Noteworthy, to date, there is no breakpoint consensus to define biocide-reduced susceptibility, including for CHG. Based on the epidemiological cutoff (ECOFF) proposed by Morrissey et al. [17], we might consider most of our isolates as non-susceptible to CHG.

A reduction of $>5 \log _{10}$ was obtained for all wild-type strains after 30-s contact with OCT at a concentration of $0.01 \%$ (=100 ppm), $0.001 \%$ (=10 ppm), and $0.0005 \%$ $(=5 \mathrm{ppm})$. OCT at a concentration of $0.0001 \%(=1 \mathrm{ppm})$ showed a time-dependent activity, achieving a reduction of $>5 \log _{10}$ after a contact time of $2.5 \mathrm{~min}$. Finally, a
Table $2 \log _{10}$ reduction factor obtained with octenidine $0.01 \%$ at a contact time of $1 \mathrm{~min}$ for all test isolates, with or without organic load

\begin{tabular}{llll}
\hline Test isolates & \multicolumn{2}{l}{$\log _{10}$ reduction factor } & \\
\cline { 2 - 4 } & Without organic load & $\begin{array}{l}\text { With albumin } 0.3 \mathrm{~g} / \mathrm{L}, \\
\text { "clean conditions" }\end{array}$ & $\begin{array}{l}\text { With albumin } \\
3 \mathrm{~g} / \mathrm{L}+\text { erythrocytes } \\
3 \mathrm{~mL} / \mathrm{L} \text {, "dirty conditions" }\end{array}$ \\
\hline E. coli (all strains) & $>5$ & $>5$ & $>5$ \\
K. pneumoniae (all strains) & $>5$ & $>5$ & $>5$ \\
E. cloacae (all strains) & $>5$ & $>5$ & $>5$ \\
A. baumannii (all strains) & $>5$ & $>5$ & $>5$ \\
P. aeruginosa (all strains) & $>5$ & $>5$ & $>5$ \\
\hline
\end{tabular}


concentration of $0.00001 \%(=0.01 \mathrm{ppm})$ was not effective for any given time lapse (Fig. 1).

All strains showed sensitivity against $0.01 \%$ OCT at a contact time of only $1 \mathrm{~min}$, even in the presence of $0.3 \mathrm{~g} / \mathrm{L} \mathrm{BSA}$ ("clean conditions") or of $3 \mathrm{~g} / \mathrm{L} \mathrm{BSA}+3 \mathrm{~mL} / \mathrm{L}$ erythrocytes ("dirty conditions"), resulting in a reduction factor of $>5 \log _{10}$ (Table 2).

\section{Conclusions}

The present study showed that octenidine dihydrochloride (OCT) is highly effective against multidrug-resistant (MR) Gram-negative (GN) pathogens within a very short period of time, under either non-organic or organic conditions, independent of increased minimum inhibitory concentrations (MICs) towards chlorhexidine digluconate $(\mathrm{CHG})$ or of the overall susceptibility to antibiotics. Among the tested isolates, some produced the most threatening resistance mechanisms that may be encountered worldwide in MRGN pathogens, namely carbapenemases.

The results obtained in this study encourage considering OCT, which is well tolerated and without resistances reported so far [18], as an alternative antiseptic for controlling the spread of MR bacteria, either being Gram-positive or Gramnegative. Further studies are required to better evaluate the impact of OCT in clinical practice for preventing infections caused by MRGN, such as that recently performed by Gastmeier et al. [19], who showed no significant impact on the prevention of MRGN acquisition in intensive care units.

Acknowledgments This study was partially supported by Schülke \& Mayr GmbH and the University of Fribourg. Active compounds were provided by Schülke \& Mayr GmbH, Norderstedt, Germany.

\section{Compliance with ethical standards}

Ethical approval Not applicable.

Conflict of interest L.P. received honoraria from Schülke \& Mayr $\mathrm{GmbH}$ as a speaker for presenting data resulting from this study in international conferences.

Informed consent Not applicable.

\section{References}

1. Laxminarayan R, Duse A, Wattal C, Zaidi AK, Wertheim HF, Sumpradit N, Vlieghe E, Hara GL, Gould IM, Goossens H, Greko C, So AD, Bigdeli M, Tomson G, Woodhouse W, Ombaka E, Peralta AQ, Qamar FN, Mir F, Kariuki S, Bhutta ZA, Coates A, Bergstrom R, Wright GD, Brown ED, Cars O (2013) Antibiotic resistance- the need for global solutions. Lancet Infect Dis 13(12):1057-1098
2. Cerceo E, Deitelzweig SB, Sherman BM, Amin AN (2016) Multidrug-resistant gram-negative bacterial infections in the hospital setting: overview, implications for clinical practice, and emerging treatment options. Microb Drug Resist 22(5):412-431

3. Potron A, Poirel L, Nordmann P (2015) Emerging broad-spectrum resistance in Pseudomonas aeruginosa and Acinetobacter baumannii: mechanisms and epidemiology. Int J Antimicrob Agents 45(6):568-585

4. Nordmann P, Poirel L (2014) The difficult-to-control spread of carbapenemase producers among Enterobacteriaceae worldwide. Clin Microbiol Infect 20(9):821-830

5. Huang SS, Septimus E, Kleinman K, Moody J, Hickok J, Avery TR, Lankiewicz J, Gombosev A, Terpstra L, Hartford F, Hayden MK, Jernigan JA, Weinstein RA, Fraser VJ, Haffenreffer K, Cui E, Kaganov RE, Lolans K, Perlin JB, Platt R; CDC Prevention Epicenters Program; AHRQ DECIDE Network and HealthcareAssociated Infections Program (2013) Targeted versus universal decolonization to prevent ICU infection. N Engl J Med 368(24): 2255-2265

6. Climo MW, Yokoe DS, Warren DK, Perl TM, Bolon M, Herwaldt LA, Weinstein RA, Sepkowitz KA, Jernigan JA, Sanogo K, Wong ES (2013) Effect of daily chlorhexidine bathing on hospitalacquired infection. N Engl J Med 368(6):533-542

7. Apisarnthanarak A, Yang Hsu L, Lim TP, Mundy LM (2014) Increase in chlorhexidine minimal inhibitory concentration of Acinetobacter baumannii clinical isolates after implementation of advanced source control. Infect Control Hosp Epidemiol 35:98-99

8. Naparstek L, Carmeli Y, Chmelnitsky I, Banin E, NavonVenezia S (2012) Reduced susceptibility to chlorhexidine among extremely-drug-resistant strains of Klebsiella pneumoniae. J Hosp Infect 81:15-19

9. Fernández-Cuenca F, Tomás M, Caballero-Moyano FJ, Bou G, Martínez-Martínez L, Vila J, Pachón J, Cisneros JM, RodríguezBaño J, Pascual Á; Spanish Group of Nosocomial Infections (GEIH) from the Spanish Society of Clinical Microbiology and Infectious Diseases (SEIMC) and the Spanish Network for Research in Infectious Diseases (REIPI); Spanish Group of Nosocomial Infections GEIH from the Spanish Society of Clinical Microbiology and Infectious Diseases SEIMC and the Spanish Network for Research in Infectious Diseases REIPI (2015) Reduced susceptibility to biocides in Acinetobacter baumannii: association with resistance to antimicrobials, epidemiological behaviour, biological cost and effect on the expression of genes encoding porins and efflux pumps. J Antimicrob Chemother 70(12):3222-3229

10. Conceição T, de Lencastre H, Aires-de-Sousa M (2016) Efficacy of octenidine against antibiotic-resistant Staphylococcus aureus epidemic clones. J Antimicrob Chemother 71(10):2991-2994

11. Goroncy-Bermes P, Brill FHH, Brill H (2013) Antimicrobial activity of wound antiseptics against extended-spectrum $\beta$-lactamaseproducing bacteria. Wound Med 1:41-43

12. Koburger T, Hübner NO, Braun M, Siebert J, Kramer A (2010) Standardized comparison of antiseptic efficacy of triclosan, PVPiodine, octenidine dihydrochloride, polyhexanide and chlorhexidine digluconate. J Antimicrob Chemother 65(8):1712-1719

13. European Committee on Antimicrobial Susceptibility Testing (EUCAST) (2016) Breakpoint tables for interpretation of MICs and zone diameters. Version 6.0. http://www.eucast.org/fileadmin/ src/media/PDFs/EUCAST_files/Breakpoint_tables/v_6.0 Breakpoint table.pdf. Accessed 7 Dec 2016

14. Robert Koch-Institut (2012) Hygienemaßnahmen bei Infektionen oder Besiedlung mit multiresistenten gramnegativen Stäbchen. Empfehlung der Kommission für Krankenhaushygiene und Infektionsprävention (KRINKO) beim Robert Koch-Institut (RKI). Bundesgesundheitsbl 55:1311-1354 
15. Clinical and Laboratory Standards Institute (CLSI) (2007) Performance standards for antimicrobial susceptibility testing; 17th informational supplement. CLSI document M100-S17. CLSI, Wayne, PA

16. British Standards Institute (2013) BS EN 13727:2012+A1:2013. Chemical disinfectants and antiseptics. Quantitative suspension test for the evaluation of fungicidal or yeasticidal activity in the medical area. Test method and requirements (phase 2, step 1), English version. European Committee for Standardization, Brussels

17. Morrissey I, Oggioni MR, Knight D, Curiao T, Coque T, Kalkanci A, Martinez JL; BIOHYPO Consortium (2014) Evaluation of epidemiological cut-off values indicates that biocide resistant subpopulations are uncommon in natural isolates of clinically-relevant microorganisms. PLoS One 9(1):e86669

18. Hübner NO, Siebert J, Kramer A (2010) Octenidine dihydrochloride, a modern antiseptic for skin, mucous membranes and wounds. Skin Pharmacol Physiol 23:244-258

19. Gastmeier P, Kämpf KP, Behnke M, Geffers C, Schwab F (2016) An observational study of the universal use of octenidine to decrease nosocomial bloodstream infections and MDR organisms. J Antimicrob Chemother 71(9):2569-2576 